\title{
Cardiovascular Symapthetic Changes In Type-II Diabetics
}

\section{K. Praveena Kumari ${ }^{* 1}$, P. Vijitha ${ }^{2}$, Nazia Farha ${ }^{2}$, M. Neeraja ${ }^{2}$.}

${ }^{* 1}$ Senior resident, A.C.S.R Government Medical College, Nellore, Andhra Pradesh, India.

${ }^{2}$ Assistant professor, A.C.S.R Government Medical College, Nellore, Andhra Pradesh, India.

\section{ABSTRACT}

Involvement of nervous system is a well-known complication of diabetes. Neuropathy is one of the most common complications of diabetes. At an early stage autonomic dysfunction may be asymptomatic or mildly symptomatic. Symptomatic autonomic neuropathy carries worst prognosis, so early diagnosis is essential for maximum benefit. This study was taken up with to assess the functional status of cardiovascular sympathetic function in normal subjects and type-II diabetics of both the sexes. Quantitative sympathetic function tests were performed in 100 subjects, 50 of them were diabetics comprising the test group and 50 were non diabetic patients as control group. Descriptive statistical analysis and Studentst- test was applied to test the significance of changes in cardiovascular sympathetic function. There was a statistically significant alternation in ANS functions in the test group when compared to control group. Sympathetic cardiovascular responses were altered significantly. Decreased blunted BP responses are associated with CAN in type II diabetes suggesting that impairment of sympathetic function. DM affects cardiovascular sympathetic function adversely through various metabolic and vascular mechanisms.

KEY WORDS: Sympathetic, Cardiovascular, Heart rate, Diabetes.

Address for correspondence: Dr. K. Praveena Kumari, Senior resident, Department of Physiology, A.C.S.R Government M edical College, Nellore, Andhra Pradesh, India.

E-Mail: dr.praveena21@gmail.com

\begin{tabular}{|c|c|}
\hline \multicolumn{2}{|r|}{ Online Access and Article Informtaion } \\
\hline \multirow{2}{*}{$\begin{array}{c}\text { Quick Response code } \\
\text { Dol: } 10.16965 \text { /ijims.2016.138 }\end{array}$} & $\begin{array}{l}\text { International Journal of Integrative Medical Sciences } \\
\text { www.imedsciences.com }\end{array}$ \\
\hline & $\begin{array}{l}\text { Received: 03-07-2016 } \\
\text { Reviewed: 03-07-2016 }\end{array}$ \\
\hline Source of Funding: Self & Conflicts of interest: None \\
\hline
\end{tabular}

\section{INTRODUCTION}

Diabetes mellitus (DM) is a global epidemic affecting 371 million people worldwide with a significant proportion (50\%) remaining undiagnosed. Prevalence of diabetes is increasing in India also. The concern is that India would be having the highest population of diabetes by 2025 and is all set to become the diabetic capital of the world. Nearly $20-30 \%$ of diabetic patients showing the impairment of autonomic function, prevalence rates, however, increased both with age (up to $44 \%$ in type-II patients aged $40-70$ years) and diabetes duration (up to $65 \%$ in type-II patients with longstanding diabetes). The available longitudinal studies indicated an annual increase in prevalence of Cardiovascular Autonomic Neuropathy CAN of about $6 \%$ in type-II diabetes. Neuropathy is one of the most common complications of diabetes. It is estimated that almost one in six people are currently at risk of developing diabetes related complication especially seen in 40 to 60 years age group [1].

Diabetes mellitus (DM) is a metabolic disorder characterized by hyperglycemia due to absolute or relative deficiency of insulin. Involvement of autonomic nervous system is a well-known complication of type-Il diabetes. The impairment of sympathetic and parasympathetic divisions of the autonomic nervous system (ANS) leads to diabetic autonomic neuropathy (DAN), a condition that may affect different organ systems 
such as cardiovascular, gastrointestinal genitourinary, sudo-motor, and visual lead to neuropathy, nephropathy, and retinopathy. Specifically, the presence of symptoms or signs suggestive of autonomic changes such as erectile dysfunction, dizziness, intermittent visual impairment, post-prandial hypotension, resting tachycardia, or exercise intolerance (dyspnea) in persons with DM [2].

One of the most over looked of all serious complications of diabetes is Cardiovascular Autonomic Neuropathy (CAN) [3]. It M ay occur when there is an impairment of an autonomic control of the cardiovascular system. At an early stage autonomic dysfunction may be asymptomatic or mildly symptomatic resulting in abnormalities in heart rate control, Silent myocardial infarction. Symptomatic autonomic neuropathy carries worst prognosis with a high risk of cardiac arrhythmias and sudden death in type II diabetes, so early diagnosis is essential for maximum benefit [4].

The integrity of autonomic nervous system is studied by measurement of changes in HR or blood pressure (BP) under various conditions such as blood pressure response to standing, blood pressure response to sustained handgrip blood pressure response to cold pressure test, heart rate response to standing, heart rate response to deep breathing and heart rate response to Valsalva maneuver. The determination of the presence of CAN is usually based on a battery of these autonomic function tests rather than just on one test. A number of autonomic function tests considered reliable, reproducible, simple and quick to carry out and all of them are non-invasive [5].

Cardiovascular autonomic neuropathy has been linked to postural hypotension, exercise intolerance, enhanced intraoperative cardiovascular lability, and increased incidence of asymptomatic ischemia, myocardial infarction, and decreased likelihood of survival after myocardial infarction. The activity of the autonomic nervous system is of crucial importance in the moment to moment regulation of heart rate and blood vessels resistance, thereby controlling arterial pressure, cardiac output and tissue perfusion. Assessment of cardiovascular autonomic nerve damage can be
Cardiovascular Symapthetic Changes In Type-Il Diabetics.

made from the combined results of simple noninvasive cardio-autonomic tests [6]. Quantitative autonomic function tests are widely used to assess autonomic function. The Deep breathing test, Valsalva maneuver, Postural tachycardia index, Orthostatic test, Isometric handgrip test and cold pressor test have been used as simple, reliable and reproducible as well as non-invasive methods of studying the cardiac autonomic function. Cardiac autonomic dysfunction indicates diffuse damage throughout the autonomic nervous system. Against this background, the present study was undertaken to evaluate cardiovascular sympathetic function tests in diabetes and match the data so obtained with healthy non diabetic individuals as controls.

\section{MATERIALS AND METHODS}

The Present prospective study was conducted in the department of physiology, Siddhartha medical college and Govt. Gen. Hospital, Vijayawada during October 2012 to September 2014. The study was undertaken to analyse the differences in cardiovascular sympathetic function tests in normal individuals and type-II diabetics in the age group 40-60 years.

Selection of Subjects: In the present study total 100 subjects were selected which includes group-A (controls) 50 normal individuals and also group-B (cases) 50 type-II diabetics who are diagnosed based on WHO criteria. They were selected from the general population of Vijayawada City randomly and patients who were attending government general hospital, Vijayawada.

Inclusion Criteria: The protocol was explained to the subjects and patients, who volunteered for the present study and informed consent was obtained from each of the participant. Includes 50 healthy, non-diabetics, non-smokers within the age group of 40-60 years. Cases of already diagnosed type-Il diabetes mellitus who are full filling who criteria's. Who are attending medicine out patient at Govt. General Hospital, Vijayawada with in the age group of $40-60$ years are selected.

Exclusion Criteria: Diagnosed diabetic patients are excluded. Subjects who are suffering from cardiac disorders are excluded as control groups. Non-diabetic patients are excluded from the 
study, Patients taking medications other than oral hypoglycemics that could influence the autonomic functions and drugs those could affect the cardiovascular functions are excluded. Patients suffering from cardiac disorders are excluded.

$M$ aterials and used were Mercury Sphygmomanometer, Hand grip dynamometer, Stethescope, Cold water.

Method of Collection of Data: The following parameters were recorded:

Physiological Parameters: The subjects were instructed not to have coffee, tea cola 12 hours before the tests and were asked to have light breakfast two hours before the tests. The subject was asked to relax in supine position for 30 minutes. B.P was measured with sphygmomanometer. The cardiovascular tests performed are detailed below in the order of execution. These tests were demonstrated to the subjects.

Resting pulse rate: The subjects / patients were asked to take rest for 10 minutes and radial pulse rate was recorded in supine position and expressed as beats / min.

Resting Blood Pressure: The resting blood pressure was recorded in supine position using mercury sphygmomanometer by the standard auscultatory Riva-Rocci method and expressed in $\mathrm{mm}$ of $\mathrm{Hg}$.

Body Temperature: The body temperature (oral) was recorded in ${ }^{\circ}$, using clinical thermometer.

Sympathetic Function Tests: The following sympathetic function tests were performed, that are B.P response to standing, response to sustained hand grip, response to cold pressure test were used for testing sympathetic function and later three that are HR response to standing, response to deep breathing test, valsalva mamouvere for testing parasympathetic nervous system.

Blood Pressure Response to Standing: The subject was asked to rest in a supine position for 5 minutes. The resting B.P was recorded. The subject was then asked to stand unaided and remain standing unsupported for 1 minute. Blood pressure was recorded in the upper arm by sphygmomanometer in supine position at rest and again recorded 1 minute after standing. The postural fall in systolic blood pressure was taken as a difference between systolic pressure in supine and systolic pressure on $1 \mathrm{~min}$ after standing.

Normal: $>10 \mathrm{mmHg}$. Borderline: 11 - $20 \mathrm{mmHg}$. Abnormal: $>30 \mathrm{mmHg}$.

Blood Pressure Response to Sustained Handgrip: Resting systolic blood pressure was recorded. Subjects / patients were asked to maintain handgrip in other arm at $30 \%$ of maximum voluntary pressure for up to 5 minutes. Blood pressure was recorded in the nonexercising arm thrice at 1-minute interval during the procedure. The maximum reading of the diastolic blood pressure was taken as the final value.

Normal: $>16 \mathrm{mmHg}$. Borderline: $11-15 \mathrm{mmHg}$. Abnormal: $>10 \mathrm{mmHg}$

Blood Pressure Response to Cold Pressor Test (CPT): The cold pressor test was evaluated by immersion of subject's left hand (up to wrist) in cold water at $10^{\circ} \mathrm{C}$ for $2 \mathrm{~min}$. in the recumbent position. Blood pressure was measured before immersion and $1 \mathrm{~min}$. after immersion of hand. In normal persons immersing of hands in ice water raises the systolic pressure by $15-20$ $\mathrm{mmHg}$ and the diastolic pressure by $10-15$ $\mathrm{mmHg}$. An elevation $>25 \mathrm{mmHg}$ for patients with "hypertension" have been viewed as predisease.

Statistical methods of the data: Descriptive statistical analysis has been carried out in the present study. Results on continuous measurements are presented as Mean and S.D.(standard deviation) of all the cardiovascular parameters Systolic Blood Pressure, Diastolic Blood Pressure were worked out, before and after evaluation of sympathetic function tests for both groups. Students - t test was applied M icrosoft Excel 2007 with P value less than 0.05 $(P \varangle 0.05)$ was considered statistically significant to test the significance of changes in cardiovascular parameters stated above and all p-values reported are two tailed. Data represented as appropriate tables, bar diagrams, for discussion under different headings.

\section{RESULTS}


K. Praveena Kumari, P. Vijitha, Nazia Farha, M. Neeraja.

Table 1: M ean, SD of age in both study groups.

\begin{tabular}{|c|c|c|c|c|c|}
\hline & & (yrs) & $t$-value & P value & Inference \\
\hline Group & Mean & S.D & \multirow{3}{*}{0.82} & \multirow{3}{*}{$>0.05$} & \multirow{3}{*}{$\begin{array}{c}\text { Non- } \\
\text { significant }\end{array}$} \\
\hline Group-A & 50.26 & 5.27 & & & \\
\hline Group-B & 49.32 & 5.83 & & & \\
\hline
\end{tabular}

Table 2: M ean, SD of FBS, PGBS, and HbA1C in both study groups.

\begin{tabular}{|c|c|c|c|c|c|}
\hline & \multicolumn{2}{|c|}{ Group - A } & \multicolumn{2}{c|}{ Group - B } & \multirow{2}{*}{ p-value } \\
\hline & MEAN & SD & MEAN & SD & \\
\hline FBS & 163.1 & 24.68 & 92.08 & 12.27 & 0 \\
\hline PGBS, & 287.24 & 29.52 & 127.24 & 9.27 & 0 \\
\hline HbA1C & 8.39 & 1.32 & 4.53 & 0.3 & 0 \\
\hline
\end{tabular}

Table 3: Mean, SD of resting pulse rate in both study groups.

\begin{tabular}{|c|c|c|c|c|c|}
\hline & \multicolumn{2}{|c|}{ RPR } & t-value & P value & Inference \\
\hline Group & Mean & S.D & & & \\
\cline { 1 - 3 } Group-A & 78.52 & 4.36 & \multirow{2}{*}{2.92} & \multirow{2}{*}{0.004} & Significant \\
\cline { 1 - 3 } Group-B & 81.4 & 5.07 & & & \\
\hline
\end{tabular}

Table 4: M ean, SD of resting blood pressure in both study groups.

\begin{tabular}{|c|c|c|c|c|c|}
\hline & \multicolumn{2}{|c|}{$\begin{array}{r}\text { Resting Blood Pressure } \\
\text { (SBP \& DBP) }\end{array}$} & \multirow{2}{*}{ t-value } & Pvalue & Inference \\
\cline { 1 - 3 } Group & Mean & SD & & & \\
\hline Group-A & 122.8 and 78 & 9.91 and 12.4 & SPB: 5.96 & SBP: $\varangle .0001$ & Significant \\
\hline Group-B & 136.2 and 82.2 & 8.81 and 9.54 & DBP: 2.29 & DBP: 0.02 & Significant \\
\hline
\end{tabular}

Table 5: Mean, SD of body temperature in both study groups.

\begin{tabular}{|c|c|c|c|c|c|}
\hline & \multicolumn{2}{|c|}{ Resting Body } & t-value & P-value & Inference \\
\hline Group & Mean & S.D & & & \\
Group-A & 97.24 & $\mathbf{0 . 5 3}$ & \multirow{2}{*}{2.66} & \multirow{2}{*}{0.009} & Significant \\
\hline Group-B & $\mathbf{9 7 . 1 5}$ & $\mathbf{0 . 4 6}$ & & & \\
\hline
\end{tabular}

Table 6: Comparison of Systolic blood pressure response to standing in group-A and group-B and results of t-test $:$ values mentioned are mean and SD in each group.

\begin{tabular}{|c|c|c|c|l|}
\hline Group & Mean \pm SD & t-value & P value & Inference \\
\hline Group-A & $118 \pm 9.6$ & \multirow{2}{*}{2.15} & 0.03 & Significant \\
\hline Group-B & $114.2 \pm 8.62$ & & & \\
\hline
\end{tabular}

Table 7: Comparison of D. B.P response to SHGT in group$A$ and group-B and results of t-test: values mentioned are mean and SD in each group.

\begin{tabular}{|c|c|c|c|c|}
\hline Group & Mean \pm SD & t-value & P-value & Inference \\
\hline group-A & $94.4 \pm 7.96$ & \multirow{2}{*}{2.21} & \multirow{2}{*}{0.03} & Significant \\
\cline { 1 - 2 } group-B & $90.8 \pm 8.51$ & & & \\
\hline
\end{tabular}

Table 8: Comparison of S.B.P response to CPT in control group and test and results of t-test: values mentioned are mean and SD in each group.

\begin{tabular}{|c|c|c|c|l|}
\hline Group & Mean \pm SD & t-value & P-value & Inference \\
\hline Group-A & $132.2 \pm 9.1$ & \multirow{2}{*}{4.03} & 0.0001 & Significant \\
\hline Group-B & $139.8 \pm 9.8$ & & & \\
\hline
\end{tabular}

Int J Intg M ed Sci 2016;3(7):353-60. ISSN 2394 - 4137
Fig 1: The graphical representation of Mean of systolic blood pressure in response to standing of both groups.

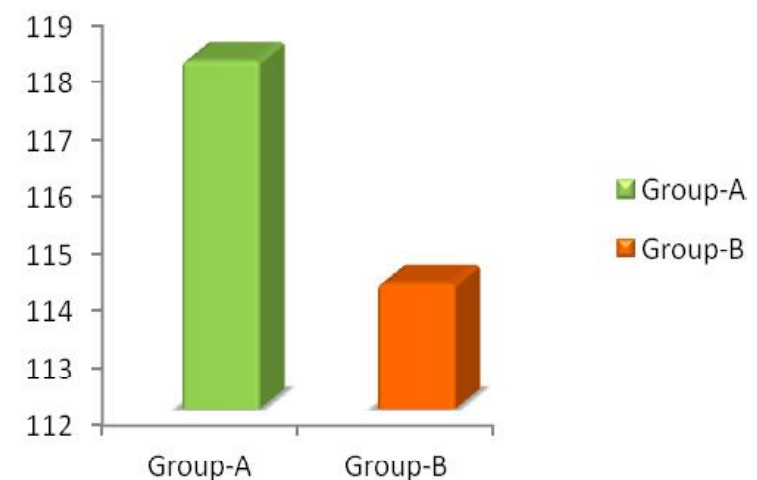

Fig. 2: The graphical representation of $M$ ean of diastolic blood pressure in response to sustained hand grip of both groups.

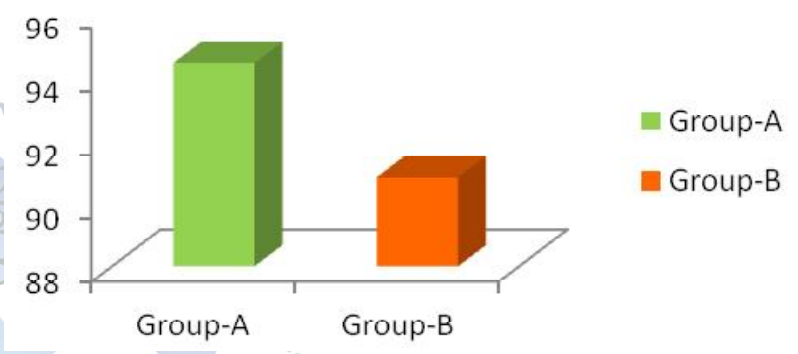

Fig 3: The graphical representation of Mean of systolic blood pressure in response to cold pressor test of both groups.

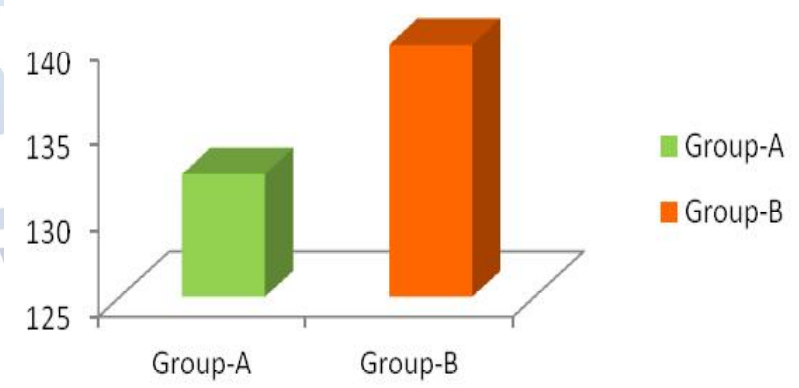

\section{DISCUSSION}

The present study was designed to assess the cardiovascular autonomic function in type II diabetics and normal subjects by cardiovascular reflex tests. These include blood pressure response to standing and sustained handgrip and cold pressor test to evaluate the sympathetic function.

Physiological Parameters: The mean \pm SD of Age of both groups were found to be group-A: $50.26 \pm 5.57$, group-B: $49.3 \pm 5.8$ respectively. There will be no significant association between age of both groups with $P>0.05$. The mean $\pm S D$ of resting pulse rate of both groups were found to be: group-A : $78.52 \pm 4.36$, group-B: $81.4 \pm$ 5.07 respectively with statistically significant 
association between both groups with $\mathrm{P} \varangle 0.05$. The mean $\pm S D$ of resting blood pressure, systolic and diastolic, of both groups were found to be in group-A : $122.8 \pm 9.91$ and $78 \pm 8.8$, group-B: 136.2. \pm 12.4 and $82.2 \pm 9.5$, respectively with statistically significant association between both groups with $\mathrm{P}<0.001$ and $\mathrm{P}<0.05$ respectively. The mean $\pm S D$ of body temperature of both groups were, group-A: 97.2 \pm 0.53 OF, group-B: $97.1 \pm 0.46{ }^{\circ} \mathrm{F}$ respectively with statistically significant association between both groups with statistically significant association between both groups with $P \varangle 0.005$.

Three sympathetic function tests were studied in both groups.

Blood Pressure response to standing: This was evaluated in 100 subjects, 50 of them were non-diabetics comprising group-A and 50 were type-2diabetics comprising group- $B$. The mean and standard deviation of group-A and group-B are shown in Table 6 . The mean and standard deviation of SBP of group-A and group-B were $118 \pm 9.6$ and $114.2 \pm 8.62$ respectively and DBP were $82.1 \pm 10.3$ and $86.8 \pm 8.7$ respectively. This study shows statistically significant difference in S.B.P. response to standing between group-A and group- $B$ with $P \varangle 0.05$. The graphical representations of Mean of B.P. response to standing test in two groups are shown in Figure - 1.

In present study, the mean systolic blood pressure was found to be decreased in both groups from lying down to one minute after standing. The fall was due to decreased venous return and decreased cardiac output on standing affected by gravity. The mean diastolic blood pressure was found to be increased The rise in diastolic blood pressure after standing is brought by increased sympathetic discharge on heart and blood vessels initiated by change in blood pressure. The heart and blood vessels are part of the cardiovascular system, which controls blood circulation. Damage to nerves in the cardiovascular system interferes with the body's ability to adjust blood pressure and heart rate. As a result, blood pressure may drop sharply after standing, causing a person to feel lightheaded or even to faint.

In normal individuals, on standing up, a fall in central venous pressure, cardiac output and blood pressure occurs, due to pooling of blood in lower extremities. The baroreceptors in the arch of aorta and carotid sinus are stimulated, sending signals via vasomotor centre to autonomic nervous system, increasing heart rate and arterial pressure through reflex vasoconstriction. Fall in systolic blood pressure is not more than $10 \mathrm{~mm}$ of $\mathrm{Hg}$. Blood pressure response to standing was significantly reduced in group- $B$ when compared to group- $A(P<0.05)$. It can thus be concluded from Table 6 and Figure - 1 that the Blood pressure response to standing, a measure of cardiac sympathetic function is reduced in group- $\mathrm{B}$. The finding of the present study is in conformity with earlier studies.

Alexandra Diana Comanescu and collegues observed that B.P response to standing, a measure of sympathetic function was found to be Borderline in $5.67 \%$ and abnormal $33.96 \%$ of cases. They found that autonomic function tests statistically decreased in patients comparatively to controls Autonomic impairment is an early and frequent finding in type-Il diabetes. CAN should be actively diagnosed beginning from its subclinical phase long before clinical signs of autonomic dysfunction become obvious. The great proportion of affected patients in the group $<$ years of diabetes duration leads to the statement that screening for CAN should be done in the moment of diagnosis in type-II diabetes [7]. AK Basu. et.al. have showed in type 2 diabetes patients were examined, B.P response to standing was found to be abnormal in $14 \%$ of cases and borderline in $22 \%$ of cases. CAN was noticed in $54 \%$ cases. Parasympathetic neuropathy was found in $52 \%$ cases and sympathetic neuropathy in $20 \%$ cases. Therefore by this study they concluded that assessment of autonomic cardiovascular reflexes affords a satisfactory method for the evaluation of CAN [8]. Bennett T, et. al., have reported a normal response to standing (little or no change in systolic blood pressure and mild tachycardia) in one category of diabetic patients, another category of patients showed initial fall in systolic blood pressure and more sustained tachycardia on standing, a third category of patients showed a definite fall in systolic blood pressure and drastic tachycardia on standing and fourth 
category showed definite fall in systolic blood pressure on standing but no appreciable tachycardia [9].

Blood Pressure response to sustained handgrip: The mean and standard deviation of group-A and group-B are shown in Table 7. The mean and standard deviation of SBP group-A and group-B were $145.5 \pm 9$ and $155.6 \pm 12.8$ respectively and DBP $94.4 \pm 8$ and $90.8 \pm 8.5$ and This study shows statistically significant difference in D.B.P. response to sustained handgrip test between group-A and group-B with $P \varangle 0.05$. The graphical representations of Mean DBP of sustained handgrip test in the two groups are shown in Figure - 2.

In the present study it was observed that, both mean systolic and diastolic blood pressures increased steadily during sustained handgrip at an interval of one minute until five minutes when compared to their resting states in normal healthy subjects of both groups. The observed graded increase in blood pressure physiologically designed to attempt to overcome the mechanical resistance of muscle blood flow so as to maintain some blood supply to the working muscle. The resting rise and rise of blood pressure during sustained handgrip in diabetes may be attributed to diabetic atherosclerosis. During sustained handgrip a sharp rise in blood pressure occurs, due to a heart rate dependent increase in cardiac output with unchanged peripheral resistance should the normal reflex pathways be damaged, as in diabetics with extensive peripheral sym pathetic abnormalities, the rise in blood pressure is abnormally small. During sustained isometric muscular exercises, like sustained handgrip, there is normally a heart rate-dependant increase in cardiac output, and a consequent increase in blood pressure. The heart rate increase is initiated by a release from vagal tone. The pressure rise could be ascribed both to heart rate acceleration and peripheral vasoconstriction.

Apart from being regulated by the ANS and endocrines, blood flow is controlled by substances released from the endothelium. NO is an important mediator of endothelium dependent vasodilation. Decreased blood flow to nerves in diabetes is associated with a decreased contribution of NO to basal vascular tone. Pathogenesis of diabetic neuropathy is considered to be both hyperglycemia induced pathologic changes intrinsic to neurons and ischemia induced neuronal damage by decreased neurovascular blood flow. Because of these vascular elements, diabetic neuropathy is considered to be a microvascular complication. There was a significant decline in DBP response to sustained handgrip in group-B when compared to group-A $(P<0.05)$. It can thus be concluded from Table 7 and Figure - 2 that the blood pressure response to sustained handgrip, a measure of cardiac sympathetic function is reduced in group-B (test group). The finding of the present study is in conformity with earlier studies.

Bandi Hari Krishna and his colleagues were found that duration of DM was negatively correlated with the autonomic function tests and by this study it is concluded that, the duration of diabetes has more effect on CAN They evaluated B.P response to sustained hang grip test, a measure of sympathetic function in all subjects and observed that decrease in B.P response to SHGT in type-II diabetics when compared with controls (mean of B.P response to SHGT in cases $6.28 \pm 2.85$ mean in controls $13.24 \pm 2.56$ ). So, it is recommended that testing for CAN should be done as a routine investigation of DM patients [10]. Md.Khairul Alam and collegues were observed that B.P response to SHGT was found to decreased in both groups of type-Il diabetics when compared to controls (mean in both groups of diabetic are $24.80 \pm 9.63,19.86 \pm 10.82$ and in controls $27.84 \pm 7.83$ ). By this study they concluded that significant deterioration of sympathetic nerve function was observed in diabetic group with longer duration ${ }^{(11)}$. Ewing. D. J. described an abnormally small blood pressure increase during sustained handgrip has been found in unselected diabetic subjects, in diabetic subjects with autonomic neuropathy [12]. Valensi P, et.al. were found 84 diabetic patients completed the handgrip test and there was an evidence of abnormal cardiac response to handgrip in 15 of these patients, who all had parasympathetic abnormalities as well [13].

\section{Blood Pressure response to cold pressor test:}

The mean and standard deviation of group-A and 
group-B are shown in Table 8 . The mean and standard deviation of SBP of group-A and groupB were $139.8 \pm 9.8$ and $132.2 \pm 9.1$ respectively and DBP were $90.8 \pm 10$ and $84.4 \pm 8.6$. The mean systolic blood pressure was found to be statistically significant increase in both groups 1 minute after the immersion when compared to before immersion. This study shows statistically significant difference in B.P. response to cold pressor test between group-A and group $B$ with $P<0.0001$. The graphical representations of M ean of cold pressor test in two groups are shown in Figure - 3 .

Blood pressure response to cold pressor test was significantly increased in group-B when compared to group-A $(P<0.0001)$. It can thus be concluded from Table 8 and Figure - 3 that the SBP response to cold pressor test, a measure of cardiac sympathetic function is increased in group-B (test group). The finding of the present study is in conformity with earlier studies.

The skin surface cooling in the cold pressor test (CPT) usually triggers generalized sympathetic activation with increase of systolic and diastolic blood pressure. The rise in both systolic and diastolic blood pressure one minute after immersion of hand (upto wrist) in $10^{\circ} \mathrm{C}$ cold water was due to vasopressor reaction induced by pain through neurogenic reflex arc. The increase in norepinephrin as a part of sympathetic stimulation may also form a part of response to cold pressor test (14) In type-II diabetes, hyperglycemia induces the formation of oxygen free radicals and protein kinase $C$. These factors interfering with endothelium-dependent vasodilation. So Hyperglycemia have been implicated in the development of vascular dysfunction in diabetes, leads to ineffective vasodilation which may manifests as sustained vasoconstriction. So that there are more increase in blood pressure in type-2 diabetics.

Nanda R. Chavan, et.al. in their study they observed that B.P response to cold pressor test was statistically significant increase in type-II diabetics compared to controls with means 1 $17.06 \pm 14.82$ and $139.82+18.51$ in controls and cases respectively [15]. I.Velchevaa, $P$. Damianova, et.al. they found that statistically significant increase with B.P response to cold pressor test in type-II diabetics compared to controls with mean SBP $124.40 \pm 15.64$ and $133.76 \pm 15.70$ in controls and type-II diabetics respectively [16].

The main finding of this study is that, decreased Heart rate variability and blunted blood pressure responses, as measured by cardiovascular testing, is associated with type II diabetes patients. Hence, it is suggested that cardiovascular autonomic functions declining in type II DM patients as the disease progresses.

\section{CONCLUSION}

The Cardiovascular autonomic neuropathy is associated with in patients with type 2 diabetes, the findings are consistent with the hypothesis that suggest multiple etiological factors leads to metabolic and vascular complications resulting in cardiovascular autonomic neuropathy in type-Il diabetes mellitus. There was a statistically significant alternation in cardiovascular sympathetic functions in the group-B (test group) when compared to group$A$ (control). Decreased blunted $B P$ responses are associated with CAN in type II diabetes suggesting that impairment of sympathetic function. DM affects cardiovascular sympathetic function adversely through various metabolic and vascular mechanisms. As the clinical importance of diabetic autonomic neuropathy is increasingly recognized, this study elucidates that simple bedside tests, as employed in this study, based on cardiovascular reflexes, can provide an objective and useful guide to know the presence and degree of damage to autonomic nervous system in diabetics. Proper treatment regimens, and stricter dietary and exercise modifications help in controlling the glycemic status. This not only helps prevent early onset of autonomic neuropathy in diabetics, but will also eventually help in reducing the symptoms in those that have diabetic autonomic neuropathy.

\section{REFERENCES}

[1]. Luiz Clemente Rolim, Jose Sergio Tomaz de Souza, and Sergio Atala Dib. Tests for Early Diagnosis of Cardiovascular Autonomic Neuropathy: Critical Analysis and Relevance. Front Endocrinol (Lausanne). 2013;4:173.

[2]. Diagnosis and Classification of Diabetes M ellitus American Diabetes Association. Diabetes Care.2014:37;1. 
[3]. Gerasimos Dimitropoulos, Abd A Tahrani, and M artin J Stevens. Cardiac autonomic neuropathy in patients with diabetes mellitus World J Diabetes. 2014:15;5(1):17-39.

[4]. Mudassir M irza and A.N.R.Lakshmi. A comparative study of heart rate variability in diabetic subjects and normal subjects; IJBAR;2012;03(08).

[5]. Oakley I, Emond L. Diabetic cardiac autonomic neuropathy and anesthetic management: review of the literature. AANA J. 2011;79(6):473-9.

[6]. Samy M. Makary, Hamdy A. Selim', Yasser M. ElWazir, Abd El-Ariz Mahmoud. Physiological Assessment of Cardiac Autonomic Reflexes in Diabetics. Suez Canal Univ M ed J, March, 1999;2:1672.

[7]. Alexandra diana comanescu. Subclinical diagnosis of cardiovascular autonomic neuropathy in type 2 diabetes: prevalence, severity, correlations with time, metabolic and vascular factors. Romanian journal of neurology; 2010:ix:1.

[8]. AK Basu, R Bandyopadhyay, S Chakrabarti, R Paul, S Santra. A Study on The Prevalence of Cardiac Autonomic Neuropathy in Type-2 Diabetes in Eastern India. JIACM 2010;11(3):190-4.

[9]. Bennett T, Hosking D.J and Hampton J.R. Cardiovascular control in diabetes mellitus. British Medical journal. 1975;2:585-7.

[10]. Bandi Hari Krishna, M allikarjuna Reddy N, Sharan B, Singh M, Sasi Kala P, Kiran Kumar C H, Uma B V, Vijetha P, Shravya Keerthi G; Cardiovascular autonomic neuropathy in diabetics: Correlation with duration of diabetes. Biomed Res- India Volume 25 Issue 3.
[11].Md.Khairul Alam Noorzahan Begum, M.H. M olla,Shelina Begum. A study of Sympathetic nerve function status in type2 diabetes-relationship with glycemic status and duration. 2008:11:1.

[12]. Ewings D.J. cardiovascular reflexex and autonomic neuropathy. Clinical Science and Molecular Medicine. 1978;55(4):321-7.

[13]. Valensi P, Huard J.P, Giroux C, Attali J.R; Factors involved in cardiac autonomic neuropathy in diabetic patients. J Diabetes complications. 1997;11(3):180-7.

[14]. David j. Ewing,Christopher N. Martyn, Robert J. Young And basil f. Clarke; The value of cardiovascular autonomic function tests: 10 years experience in diabetes; diabetes care, septemberoctober 1985:8:5.

[15]. Nanda R. Chavan, Salim A. Dhundasi and Kusal K. Das; Determination of sensitivity among various cardiovascular autonomic function tests in diabetic patients of Bijapur. Journal of Basic \& Clinical Physiology \& Pharmacology. 2009;20:2.

[16]. I.Velchevaa, P. Damianova, St. Mantarovab, N. Antonovac; Cold pressor test: Effects on cardiac autonomic control and cerebral hemodynamic response in patients with diabetes mellitus type 2 : Series on Biomechanics. 2012:27(1-2);64-9.

\section{How to cite this article:}

K. Praveena Kumari, P. Vijitha, Nazia Farha, M. Neeraja. Cardiovascular Symapthetic Changes In Type-II Diabetics. Int J Intg M ed Sci 2016;3(7):353360. Dol: 10.16965/ijims.2016.138 